\title{
Adaptação e evidências de validade da Bateria de Personalidade Prosocial no Brasil
}

\author{
André L. A. Rabelo - Universidade de Brasília, Brasilia/DF, Brasil \\ Ronaldo Pilati - Universidade de Brasilia, Brasilia/ DF, Brasil
}

\begin{abstract}
Resumo
$\mathrm{O}$ interesse da psicologia social e da personalidade pela disposição duradoura a agir prosocialmente resultou no desenvolvimento de instrumentos capazes de medir tal "bondade." O objetivo do presente estudo foi traduzir para o português, adaptar e avaliar as propriedades psicométricas da Bateria de Personalidade Prosocial (Prosocial Personality Battery), um instrumento desenvolvido para medir diferenças individuais na disposição de agir prosocialmente. Dois estudos foram conduzidos para testar a estrutura teórica do instrumento e produzir evidências de validade. No Estudo 1, o instrumento original foi traduzido usando o método tradução-retradução. A estrutura original foi replicada e os indicadores psicométricos observados foram próximos dos relatados originalmente. No Estudo 2, a estrutura foi novamente replicada e a análise fatorial confirmatória demonstrou que, embora ainda possam haver aprimoramentos futuros, ele replica a estrutura original e pode ser usado no Brasil para estudar o comportamento prosocial.

Palavras-chave: Psicologia Social; Comportamento prosocial; Personalidade; Diferenças individuais.
\end{abstract}

Adaptation and evidence of validity of the Prosocial Personality Battery in Brazil

\begin{abstract}
The interest of personality and social psychology for the enduring disposition to act prosocially resulted in the development of instruments capable of measuring such "goodness". The aim of the present study was to translate into Portuguese, adapt and evaluate the psychometric properties of the Prosocial Personality Battery, an instrument developed to measure individual differences in the disposition to act prosocially. Two studies were conducted to test the theoretical structure of the instrument and produce evidence of validity. In Study 1, the original instrument was translated using the back-translation method. The original structure was replicated and the psychometric properties observed were similar to the originally obtained. In Study 2, the structure was replicated again and the confirmatory factor analysis showed that, even though future improvements can be made, it replicates the original structure an can be used in Brazil to study prosocial behavior.

Keywords: Social psychology; Prosocial behavior; Personality; Individual differences.
\end{abstract}

Adaptación y evidencia de validez de la Batería de Personalidad Prosocial en Brasil

\section{Resumen}

El interés de la psicología social y de la personalidad en la tendencia a actuar prosocialmente resultó en el desarrollo de instrumentos que permitan medir esa "bondad". El objetivo de este estudio fue traducir al portugués, adaptar y conocer las propiedades psicométricas de la Batería de Personalidad Prosocial (Prosocial Personality Battery), un instrumento desarrollado para medir las diferencias individuales en la voluntad para actuar prosocialmente. Se realizaron dos estudios para probar la estructura teórica del instrumento y producir pruebas de validez. En el Estudio 1, el instrumento original fue traducido por el método de traducción- retraducción. La estructura original fue replicada y los indicadores psicométricos observados fueron similares a los obtenidos originalmente. En el Estudio 2, la estructura se repitió y el análisis factorial confirmatorio mostró que, aunque el instrumento pueda ser mejorado en futuras investigaciones, reproduce la estructura original y puede ser utilizado en Brasil para estudiar el comportamiento prosocial.

Palabras clave: Psicología social; Comportamiento prosocial; Personalidad; Diferencias individuales.

Em diferentes culturas, pessoas muito generosas ao longo de suas vidas se tornaram mundialmente conhecidas e admiradas, tais como a Madre Teresa de Calcutá. Pessoas como ela demonstram, ao longo de suas vidas, uma tendência frequente e estável de ajudar outras pessoas, algo a que podemos nos referir como uma "bondade duradoura" (Penner \& Orom, 2010). O interesse da psicologia social e da personalidade por esta aparente disposição duradoura a agir prosocialmente resultou em uma vasta linha de pesquisa buscando compreender porque alguns indivíduos ajudam mais que outros, mesmo que em situações semelhantes. Um dos passos mais importantes para estudar esse fenômeno foi a construção de instrumentos capazes de medir tal Disponivel em www.scielo.br "bondade", e esse desenvolvimento metodológico ofereceu uma oportunidade para muitos avanços nesta linha de pesquisa. No intuito de melhor investigar este fenômeno, o objetivo do presente estudo foi traduzir, adaptar e avaliar as propriedades psicométricas da Bateria de Personalidade Prosocial (Prosocial Personality Battery) para o português, um instrumento desenvolvido para medir diferenças individuais na disposição a agir prosocialmente.

\section{Diferenças individuais na prosocialidade}

$\mathrm{Na}$ psicologia social e da personalidade, diversos estudos têm indicado a importância de considerar as diferenças individuais concernentes à disposição prosocial na investigação do comportamento prosocial 
(Carlo, Eisenberg, Troyer, Switzer \& Speer, 1991; Dovidio, Piliavin, Schroeder \& Penner, 2006; Penner \& Finkelstein, 1998; Penner \& Orom, 2010; Perugini, Conner \& O'Gorman, 2011; Rabelo, Hees \& Pilati, 2012; Van Lange, Otten, De Bruin \& Joireman, 1997). Graziano, Habashi, Sheese e Tobin (2007), por exemplo, encontraram que um maior nível relatado de agradabilidade (agreeableness) dos participantes, um dos traços de personalidade do Big Five, se relacionou com uma maior oferta de ajuda a pessoas em diferentes contextos e um menor viés a ajudar indivíduos com os quais os participantes possuíssem algum parentesco, enquanto indivíduos com um menor nível de agradabilidade ofereceram menos ajuda em média e tiveram um maior viés de ajudar pessoas com as quais possuíssem algum parentesco. $\mathrm{Na}$ mesma direção, Caprara, Alessandri e Eisenberg (2012) demonstraram que variáveis disposicionais como a agradabilidade, os valores de autotranscedência e as crenças de autoeficácia empática dos participantes se relacionaram com uma maior tendência a agir prosocialmente. Esses autores mediram as relações entre essas variáveis quando os participantes tinham, em média, 21 anos e depois mediram novamente, quando eles tinham, em média, 25 anos. O padrão de relação entre as variáveis se manteve semelhante, dando evidências da estabilidade temporal existente entre tais variáveis.

Outros estudos demonstraram que diferenças individuais relacionadas à prosocialidade são estáveis ao longo do tempo (Eisenberg \& cols., 2002) e são capazes de predizer significativamente diversos tipos de comportamento prosocial, tais como ajudar outras pessoas, ajudar colegas de trabalho e se envolver com diversos tipos de trabalho voluntário (Penner, 2002; Penner, Dovidio, Piliavin, \& Schroeder, 2005). Além disso, diferenças individuais na prosocialidade podem atuar como importantes variáveis moderadoras da influência de processos sócio cognitivos no comportamento prosocial (Perugini \& cols., 2011; Perugini \& Prestwich, 2007; Rabelo \& cols., 2012). Kerekes, Bereczkei e Birkas (2010), por exemplo, identificaram que traços de personalidade prosocial foram preditores do engajamento em atividades prosociais independentemente de fatores situacionais manipulados no estudo, tais como a presença de outras pessoas e o custo da ajuda. Esse conjunto de evidências reforça a ideia de que variáveis disposicionais, tais como agradabilidade, personalidade prosocial e valores de autotranscedência, são preditoras relevantes do comportamento prosocial e, além disso, podem moderar o efeito que variáveis situacionais exercem nesse tipo de comportamento.

Alguns autores buscaram desenvolver uma definição mais precisa dessa variável disposicional e instrumentos que permitissem mensurá-la. Penner, Fritzsche, Craiger e Freifeld (1995) descreveram a orientação de personalidade prosocial como uma tendência estável de pensar no bem-estar e no direito de outras pessoas, de se preocupar e ter empatia pelos outros e de agir de maneira a beneficiá-las. Penner e cols. desenvolveram e validaram um instrumento para medir essa diferença individual, a Bateria de Personalidade Prosocial (Prosocial Personality Battery), referida a partir daqui como BPP. O instrumento foi construído a partir da identificação na literatura de diferentes escalas que se correlacionassem com comportamento prosocial e que possuíssem uma fundamentação teórica que explicitasse por que os construtos medidos pela escala deveriam se correlacionar com o comportamento prosocial (Penner \& cols., 1995). Os autores realizaram uma série de estudos visando produzir evidências de validade a partir de uma bateria com 128 itens. Por conta do tamanho da bateria, os autores também desejavam diminuir o tamanho da mesma. Sucessivas coletas com amostras independentes permitiram que os autores reduzissem o número de itens da bateria para 56, inicialmente, e para 30 , em sua versão final e recomendada para uso (Penner, n.d., 2002).

Esses autores propõem que a personalidade prosocial possui duas dimensões de segunda ordem (a) empatia orientada aos outros, que organiza sentimentos e pensamentos prosociais dirigidos a outras pessoas; (b) e prestatividade, que organiza comportamentos autorrelatados que demonstram ajuda e cuidado de outras pessoas. Dependendo da amostra (e.g. estudantes de graduação, trabalhadores voluntários, trabalhadores varejistas), já foram identificadas correlações de 0,25 a 0,50 entre essas duas dimensões (Penner, 2002). Tais dimensões se estruturam em sete fatores de primeira ordem: responsabilidade social (7 itens), preocupação empática (4 itens), tomada de perspectiva (5 itens), raciocínio orientado aos outros (3 itens), raciocínio moral mútuo (3 itens) - constituindo a $1^{\text {a }}$ dimensão -, desconforto pessoal (3 itens) e altruísmo autorrelatado (5 itens) - constituindo a $2^{\mathrm{a}}$ dimensão. Em um amplo estudo sobre voluntariado utilizando a BPP como medida de variável disposicional, Penner (2002) relata que pessoas com maior endosso às dimensões da BPP são mais propensas a se envolver em diversos trabalhos voluntários, com um número maior de horas doadas para a atividade e por um maior tempo de envolvimento. Tais relações demonstram a validade preditiva da BPP como instrumento para aferir essa disposição, que é uma preditora importante do comportamento prosocial. 
Considerando a atual ênfase da literatura internacional na exploração de variáveis moderadoras e mediadoras para compreender fenômenos sociais, tais como o comportamento prosocial, e a importância que a literatura tem identificado nessa diferença individual para compreender e predizer diversos tipos de comportamento prosocial, considera-se relevante o esforço em adaptar esse instrumento para a cultura brasileira, permitindo seu uso em pesquisas de âmbito nacional. Tal esforço pode permitir, inclusive, conhecer diferenças culturais referentes às características definidoras da prosocialidade no Brasil, na medida em que um instrumento que tem sido aplicado em diversas culturas possua uma versão nacional para coleta de dados. Esta versão auxiliará na pesquisa transcultural sobre o tema, principalmente quando se considera que existem evidências de que as características específicas deste fenômeno variam culturalmente (Dovidio \& cols., 2006; Levine, Norenzayan \& Philbrick, 2001; Penner \& cols., 2005) e entre homens e mulheres (Sampaio \& Menezes, 2011).

Não foi identificada na literatura nenhuma tentativa de adaptar e produzir evidências de validade da BPP para o Brasil (foram consultadas as seguintes bases: Scielo, PePSIC, Google Scholar, Portal da CAPES). Também não foram identificados estudos específicos de adaptação para outras culturas, mas a BPP tem sido utilizada em estudos realizados em diferentes países (Finkelstein, Penner \& Brannick, 2005; Kulik, 2007; Penner \& Finkelstein, 1998; Perugini \& cols., 2011; Rabelo \& cols., 2012). Cabe ainda apontar que a despeito de tais evidências, a BPP, por ser um instrumento de autorrelato, apresenta os problemas inerentes a esse tipo de medida (Nisbett \& Wilson, 1977; Nosek, Hawkins \& Frazier, 2011; Paulhus, 1984), além de problemas de análise estatística, como a excessiva valorização por parte dos pesquisadores da área de correlações e predições estatisticamente significativas em detrimento, por exemplo, da avaliação dos tamanhos de efeito envolvidos em tais relações (Cohen, 1994; Kelley \& Preacher, 2012; Wagenmakers, Wetzels, Borsboom, van der Maas \& Maas, 2011).

Com o objetivo de adaptar a BPP e avaliar as suas qualidades psicométricas, foram conduzidos dois estudos com duas amostras independentes, relatados no presente artigo. $O$ Estudo 1 visou avaliar as qualidades psicométricas da BPP e verificar se a estrutura original de Penner \& cols. (1995) poderia ser replicada na cultura brasileira, por meio de uma análise fatorial exploratória. O Estudo 2 teve como objetivo replicar a estrutura encontrada no Estudo 1 e testar de forma mais rigorosa, a estabilidade da estrutura teórica de Penner e cols., inclusive avaliando as duas dimensões de segunda ordem que agrupariam os sete fatores da BPP, por meio de uma análise fatorial confirmatória. Ambos os estudos também avaliaram se havia diferenças de sexo quanto às dimensões da BPP.

\section{Estudo 1}

\section{Método}

Participantes

O Estudo 1 contou com 556 participantes (171 homens, 341 mulheres e 44 não informado), que aceitaram responder a um questionário pela internet. A média de idade dos participantes era de 33,75 anos $(D P=11,02)$. Quanto ao nível de escolaridade, 149 participantes relataram possuir ensino superior incompleto, 133 relataram possuir ensino superior completo, 216 relataram possuir pós-graduação, 14 relataram possuir ensino médio completo ou incompleto e 44 não relataram o seu nível de escolaridade.

\section{Instrumentos e materiais}

A tradução do instrumento foi feita por um pesquisador de origem irlandesa que vivia no Brasil há aproximadamente quatro anos. Foi utilizada a técnica "tradução - retradução" (Hambleton \& Zenisky, 2011), que consiste na produção de uma versão em português do texto original da BPP. Essa versão foi apresentada ao tradutor que a traduziu do português para o inglês, possibilitando a comparação com o texto original (Beú, 2010). A partir da comparação, foram realizados poucos ajustes na tradução, tais como o uso de palavras mais comuns no português, para facilitar a compreensão, mas que tivessem significado equivalente no inglês (e.g. a expressão put myself in their shoes do item "When I'm upset at someone, I usually try to 'put myself in their shoes' for a while" foi traduzida e adaptada da seguinte maneira: "Quando estou chateado com alguém, geralmente procuro me colocar em seu lugar"). A maioria das divergências identificadas foi da mesma natureza. A versão em português foi submetida à avaliação semântica por um grupo de juízes especialistas em adaptação de medidas, que ofereceram feedback favorável quanto à compreensibilidade dos itens. O instrumento original possui trinta itens que avaliam traços disposicionais de personalidade relacionados à maior tendência de ajudar, associados a uma escala Likert de concordância de cinco pontos, com alfas de Cronbach de ambas as dimensões superiores a 0,80 , cargas fatoriais superiores a $0,30 \mathrm{e}$ estrutura fatorial semelhante para ambos os sexos (Penner \& cols., 1995). A versão original do instrumento é composta pelos fatores: responsabilidade social - RS $(\alpha=0,65)$; preocupação empática - PE $(\alpha=0,67)$; tomada de perspectiva - TP $(\alpha=0,66)$; 
desconforto pessoal - DP $(\alpha=0,77)$; razões morais mútuas - RMM $(\alpha=0,64)$; razões relacionadas ao outro - RRO $(\alpha=0,77)$; e altruísmo autorrelatado - AAR $(\alpha=0,73)$.

Conforme descrito por Penner \& cols. (1995), o fator RS descreve uma tendência a assumir responsabilidade pelas consequências das suas ações (e.g. Eu me sentiria menos incomodado ao deixar lixo em um parque sujo do que em um parque limpo); o fator PE está relacionado com uma tendência a sentimentos voltados para os outros, como simpatia e preocupação com os outros (e.g. A miséria dos outros geralmente não me incomoda muito); o fator TP avalia a tendência a adotar a perspectiva de outra pessoa (e.g. Quando estou chateado com alguém, geralmente procuro me colocar em seu lugar); o fator DP se relaciona com a propensão a sentimentos de ansiedade e mal-estar voltados para si diante de uma situação interpessoal tensa (e.g. Quando vejo alguém que precisa muito de ajuda em uma situação de emergência, eu fico perdido); o fator RMM agrupa itens relacionados a uma tendência a levar em conta o interesse de todos envolvidos ao tomar decisões morais (e.g. Eu escolho alternativas com a intenção de satisfazer as necessidades de todo mundo); o fator RRO está associado à tendência de se focar no interesse de todos ao tomar decisões morais (e.g. Minhas decisões são geralmente baseadas na minha preocupação com outras pessoas); e o fator AAR se relaciona com uma tendência a ajudar pessoas necessitadas (e.g. Eu já ajudei a carregar os pertences de um estranho (como livros, pacotes, etc.). O conjunto de trinta itens pode ser visualizado no Anexo 1.

Conforme descrito por Penner \& cols. (1995) e Penner e Orom (2010) e como já foi descrito na revisão do presente artigo, a BPP foi construída a partir dos itens de diversas escalas relacionadas a diferentes dimensões de prosocialidade, tais como a empatia e o raciocínio moral. A partir de um grande banco de itens, os pesquisadores realizaram estudos e selecionaram os itens com melhores qualidades psicométricas para cada dimensão, resultando em uma bateria com trinta itens. Por conta da maneira como essa bateria foi originalmente elaborada, os autores analisaram os dados obtidos separadamente para cada escala e para as duas dimensões principais, e foi este o procedimento adotado no presente artigo, visando replicar os procedimentos de análise de dados e permitir um maior nível de comparabilidade entre os resultados aqui relatados e os resultados do estudo original de Penner e cols. Por último foi elaborado um questionário de dados sociodemográficos (e.g. sexo, idade).

\section{Procedimentos de coleta e Análise de dados}

Esta pesquisa foi avaliada e aprovada por um Comitê de Ética. Os dados da BPP foram coletados por meio do programa EFS Survey ("EFS survey," 2012). Esse programa permite a realização completa de pesquisas pela internet. $\mathrm{O}$ procedimento da pesquisa envolveu o envio, por meio desse programa de convites para participação na pesquisa via e-mail pessoal. No e-mail, havia informações acerca da pesquisa e o link para acessar o questionário on-line da pesquisa. Quando o participante clicava no link, abriase uma janela com o termo de livre consentimento, assim como também era apresentada a informação de que a pesquisa era anônima e que o participante poderia interrompê-la a qualquer momento sem nenhum prejuízo para ele. Caso o participante aceitasse participar, pedia-se que ele respondesse aos itens da BPP e fornecesse dados sociodemográficos. Por fim, era exibida a mensagem de agradecimento e o e-mail de um dos pesquisadores responsáveis para tirar eventuais dúvidas. A estrutura da BPP foi analisada inicialmente por meio de análise de componentes principais, da inspeção visual do gráfico de sedimentação (scree plot) e de análise paralela, visando explorar a estrutura fatorial a ser testada na análise fatorial. Também foram realizados testes de normalidade dos dados e outliers para averiguar se os pressupostos da análise fatorial exploratória estavam sendo atendidos. Por último, foram realizadas análises de variância (ANOVA) e correlações para averiguar como as dimensões da BPP se relacionariam com variáveis demográficas.

\section{Resultados e Discussão}

A análise de componentes principais indicou, por meio do critério de valor próprio superior a um, uma estrutura de oito componentes, embora o ganho de variância explicada ao adicionar um $8^{\circ}$ fator seja muito pequeno (i.e. apenas $0,03 \%$ ). O gráfico de sedimentação demonstrou que, a partir do $7^{\circ}$ fator, a adição de mais fatores resulta em um ganho baixo e homogêneo na variância explicada (ver Figura 1). Por fim, a análise paralela, realizada por meio do programa RanEigein, indicou que a variância explicada a partir do $6^{\circ}$ componente estimado tinha um valor superior do que a variância explicada pelo $6^{\circ}$ componente gerado a partir da amostra do Estudo 1. A avaliação conjunta dos resultados do gráfico de sedimentação e da análise paralela indica a possibilidade de extração de seis ou sete fatores.

Psico-USF, Bragança Paulista, v. 18, n. 3, p. 455-468, set/dez.2013 


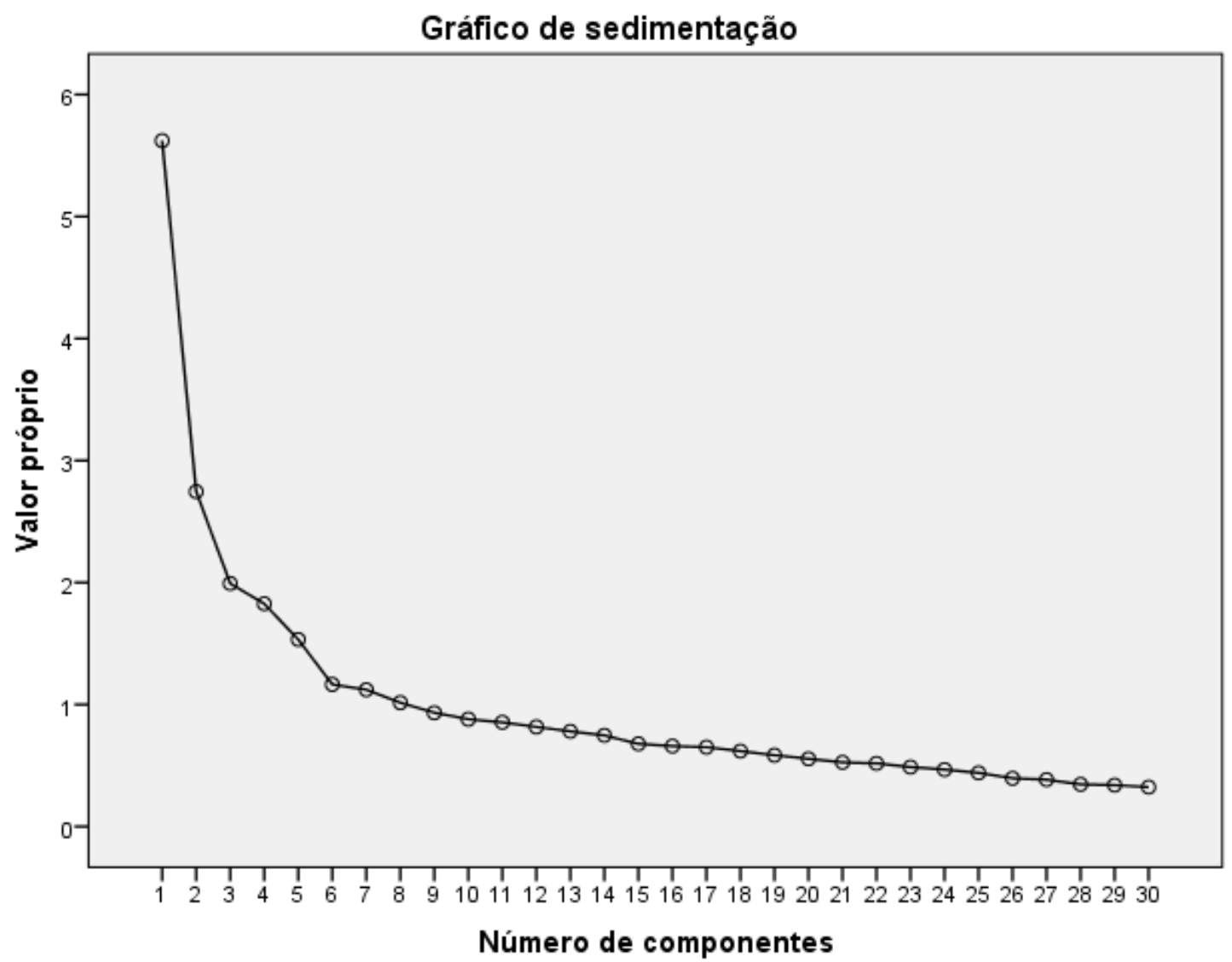

Figura 1. Gráfico de sedimentação da BPP no Estudo 1

Considerando em conjunto essas duas fontes de informação (i.e. análise do gráfico de sedimentação e análise paralela) e a estrutura original proposta pelos autores, definiu-se que a estrutura de sete fatores é uma solução adequada, garantindo, assim, a comparabilidade dessa versão brasileira com a solução fatorial de Penner (2002) e Penner e cols. (1995). Ainda que essa decisão de extração de fatores não seja ortodoxa como procedimento decisório, considera-se que a estrutura de sete fatores é razoável, tendo em conta os indicadores de extração relatados. Isso levou à decisão de analisar cada um dos sete fatores independentemente, o que permite o teste da estrutura conforme relatado pelos autores originais. Cabe argumentar que em estudos de adaptação de instrumentos para outras culturas é extremamente importante a tentativa de manter estruturas psicométricas compatíveis, pois, assim, são possíveis comparações mais precisas entre amostras de países diferentes. Isso leva a um ganho argumentativo quando a meta é a veiculação internacional da pesquisa feita no Brasil.

Então, foi realizada uma análise fatorial exploratória separadamente para os itens de cada um dos sete fatores, seguindo a estrutura relatada por Penner e cols. (1995) e Penner (2002). Apenas o item 3 foi eliminado, considerando que a análise de componentes indicou que a sua carga fatorial foi inferior a 0,30. Na Tabela 1, é possível observar os alfas de Cronbach dos fatores, as cargas fatoriais dos itens, a comunalidade de cada item e os dados relacionados à variância explicada de cada fator. 
Tabela 1. Cargas fatoriais e comunalidades dos sete fatores da BPP obtidos na AFE

\begin{tabular}{|c|c|c|c|c|c|}
\hline Responsabilidade social & Carga & $\mathrm{h}^{2}$ & Preocupação empática & Carga & $\mathrm{h}^{2}$ \\
\hline Alfa de Cronbach & 0,65 & & Alfa de Cronbach & 0,50 & \\
\hline Variância explicada (\%) & 24,33 & & Variância explicada (\%) & 22,20 & \\
\hline Item $6^{*}$ & 0,62 & 0,38 & Item 11 & 0,65 & 0,43 \\
\hline Item 1 & 0,59 & 0,35 & Item 13 & 0,44 & 0,19 \\
\hline Item 2 & 0,50 & 0,25 & Item 15 & $-0,40$ & 0,16 \\
\hline Item 7 & 0,43 & 0,19 & Item 9 & $-0,33$ & 0,11 \\
\hline Item 4 & 0,38 & 0,15 & & & \\
\hline Item 5 & 0,38 & 0,14 & & & \\
\hline Tomada de perspectiva & Carga & $\mathrm{h}^{2}$ & Desconforto pessoal & Carga & $\mathrm{h}^{2}$ \\
\hline Alfa de Cronbach & 0,63 & & Alfa de Cronbach & 0,75 & \\
\hline Variância explicada (\%) & 27,07 & & Variância explicada (\%) & 52,09 & \\
\hline Item 16 & 0,59 & 0,35 & Item 17 & 0,82 & 0,67 \\
\hline Item 10 & 0,56 & 0,31 & Item 19 & 0,75 & 0,56 \\
\hline Item 18 & 0,50 & 0,25 & Item 14 & $-0,57$ & 0,33 \\
\hline Item 8 & $-0,48$ & 0,23 & & & \\
\hline Item 12 & $-0,45$ & 0,20 & & & \\
\hline Raciocínio moral mútuo & Carga & $\mathrm{h}^{2}$ & Raciocínio relacionado ao outro & Carga & $\mathrm{h}^{2}$ \\
\hline Alfa de Cronbach & 0,58 & & Alfa de Cronbach & 0,77 & \\
\hline Variância explicada (\%) & 41,98 & & Variância explicada (\%) & 53,62 & \\
\hline Item 24 & 0,94 & 0,89 & Item 25 & 0,81 & 0,65 \\
\hline Item 22 & 0,43 & 0,19 & Item 23 & 0,76 & 0,58 \\
\hline Item 21 & 0,42 & 0,18 & Item 20 & 0,61 & 0,38 \\
\hline Altruísmo autorrelatado & Carga & $\mathrm{h}^{2}$ & & & \\
\hline Alfa de Cronbach & 0,74 & & & & \\
\hline Variância explicada (\%) & 40,48 & & & & \\
\hline Item 26 & 0,72 & 0,52 & & & \\
\hline Item 27 & 0,71 & 0,50 & & & \\
\hline Item 30 & 0,65 & 0,42 & & & \\
\hline Item 28 & 0,59 & 0,35 & & & \\
\hline Item 29 & 0,48 & 0,23 & & & \\
\hline
\end{tabular}

${ }^{*} \mathrm{O}$ número do item corresponde ao item da BPP integralmente apresentado no Anexo 1.

A análise fatorial exploratória indicou que a estrutura de sete fatores possui índices psicométricos aceitáveis e próximos aos reportados na versão original da BPP, replicando os achados da validação original. Esses dados indicam que a estrutura conceitual da BPP, apesar de ter sido desenvolvida, testada e usada primordialmente na cultura norte-americana, é adequada para estudar diferenças individuais da prosocialidade no Brasil. Entretanto, os índices psicométricos observados neste estudo, bem como os índices do instrumento original, não são totalmente satisfatórios, o que diminui a probabilidade de que tal estrutura possa ser replicada em estudos futuros (LeBel \& Paunonen, 2011). As evidências encontradas no Estudo 1 se somam às evidências de que a BPP pode ser adaptada a diferentes culturas com diferentes línguas (Kulik, 2007; Perugini \& cols., 2011) e apresentar evidências de validade semelhantes às que os Psico-USF, Bragança Paulista, v. 18, n. 3, p. 455-468, set/dez 2013 
autores que criaram o instrumento original relataram (Penner \& cols., 1995).

Também foi realizada uma análise tendo os escores dos sete fatores de primeira ordem como base da matriz de correlações para o teste da extração dos fatores de segunda ordem. O mesmo procedimento para teste da extração de fatores foi realizado para definir a extração da segunda ordem. Os resultados indicaram apenas dois componentes com autovalores acima de um; a análise do gráfico de sedimentação indicou que, a partir da adição de um terceiro fator, pouca variância é explicada adicionalmente; e a análise paralela apontou que o segundo componente, extraído a partir da amostra, possuía um valor de variância explicada superior ao valor do segundo componente extraído por estimação. Entretanto, quando essa estrutura de segunda ordem foi submetida a uma análise fatorial com extração forçada de dois fatores, o resultado não corroborou exatamente o preconizado pelos autores da BPP.

Essa análise, com rotação varimax, indicou $\mathrm{O}$ seguinte agrupamento entre os sete fatores - em uma dimensão, se agruparam as dimensões: RRO, com carga fatorial de 0,86 ; RMM, com carga fatorial de 0,72 ; PE, com carga fatorial de $-0,39$; TP, com carga fatorial de 0,34 ; e, na outra dimensão: TP, com carga fatorial de 0,60 ; RS, com carga fatorial de -0,57; DP, com carga fatorial de -0,40; AAR, com carga fatorial de 0,43. Como pode ser observado, os sete fatores não se organizaram da mesma maneira que Penner e cols. (1995) haviam identificado, quando, por exemplo, o fator TP apresentou cargas fatoriais acima de $0,30 \mathrm{em}$ ambas as dimensões, o que representa uma possível fragilidade quanto à replicabilidade dessa dimensão na cultura brasileira. Penner e cols. (1995) e Penner (2002) identificaram que a dimensão de empatia orientada ao outro deveria agrupar os fatores RS, PE, TP, RMM e RRO, enquanto a outra dimensão de prestatividade agruparia os fatores DP e AAR. Além do que já foi comentado sobre o fator TP, o fator RS não se agrupou com os fatores esperados.

Por último, foi identificada uma diferença de sexo quanto às dimensões de PE e DP: quanto à PE, homens exibiram, em média, escores maiores $(M=1,98$; $D P=0,69)$ do que as mulheres $(M=1,76 ; D P=0,55), F$ $(1,510)=14,43 ; p<0,001 ; \eta_{\mathrm{p}}^{2}=0,03$; e quanto à DP, homens exibiram escores médios menores $(M=2,13$; $D P=0,75)$ do que as mulheres $(M=2,32 ; D P=0,82), F$ $(1,510)=6,63 ; \quad p=0,010 ; \quad \eta_{\mathrm{p}}{ }^{2}=0,01$. Os resultados relacionados às diferenças de sexo nessas dimensões de segunda ordem são, em parte, inconsistentes com a literatura. Estudos anteriores com amostras brasileiras utilizando o Interpersonal Reactivity Index, outro instrumento para a avaliação de construtos teóricos correlatos à prosocialidade, indicaram que mulheres apresentam maiores níveis de preocupação/consideração empática e de desconforto/aflição pessoal, duas dimensões que são comuns à BPP (Sampaio \& Menezes, 2011). No presente estudo, os homens apresentaram níveis significativamente maiores de PE, o que pode indicar diferenças culturais dentro do próprio Brasil que merecem melhor exploração em pesquisas futuras. Com o intuito de avaliar de maneira mais rigorosa e tentar replicar a estrutura original do instrumento, bem como os achados relacionados às diferenças de sexo, foi realizado o Estudo 2.

\section{Estudo 2}

\section{Participantes}

O Estudo 2 contou com 557 participantes. Considerando que o método de Maximum Likelihood (MLE) adotado na análise fatorial confirmatória deste estudo demanda um banco sem casos omissos para ser realizado e que o número de participantes com casos omissos era pequeno em comparação com o tamanho total da amostra, optou-se por excluir participantes com casos omissos. A amostra final do estudo contou com 543 participantes (204 homens, 309 mulheres e 30 não informado) que aceitaram participar de uma pesquisa pela internet. A média de idade dos participantes era de 34,49 anos $(D P=11,14)$. O nível de escolaridade da amostra usada no Estudo 2 se distribuiu da seguinte maneira: ensino superior incompleto $(\mathrm{n}=162)$; ensino superior completo $(\mathrm{n}=120)$; pós-graduação $(\mathrm{n}=218) ; \quad$ ensino médio completo ou incompleto ( $\mathrm{n}=13)$; não informaram $(\mathrm{n}=30)$.

\section{Instrumentos e materiais}

No Estudo 2, foram usados os mesmos instrumentos e materiais usados no Estudo 1.

\section{Procedimentos de coleta e Análise de dados}

De maneira semelhante ao Estudo 1, os dados foram coletados por meio do EFS Survey. O conteúdo e a ordem de apresentação dos instrumentos foi rigorosamente igual à do Estudo 1. A análise dos dados foi feita por meio do programa $A M O S \AA$. Foi realizada uma análise fatorial confirmatória separada para cada um dos sete fatores da BPP utilizando o MLE. Também foi usado o procedimento de reamostragem bootstrapping, de forma a avaliar se haveria estabilidade na distribuição dos parâmetros a despeito da falta de normalidade multivariada (Byrne, 2010; Pilati \& Laros, 2007). Julgou-se que tal procedimento era adequado 
para o teste de estabilidade das estimações, considerando-se o tamanho da amostra, que não habilitava o uso de outros métodos de estimação diferentes do MLE. Por fim, foi realizada uma análise confirmatória para testar a estrutura de duas dimensões de segunda ordem que organizariam os sete fatores da $\mathrm{BPP}$, visando testar a estrutura original proposta pelos autores.

\section{Resultados e Discussão}

Os resultados da análise fatorial confirmatória são exibidos na Tabela 2. Como pode ser observado, os índices psicométricos permaneceram, de maneira geral, semelhantes aos obtidos no Estudo 1. O teste de bootstrapping indicou que as médias estimadas de sub amostras criadas a partir dos dados originais eram próximas das médias obtidas na amostra original na maioria dos itens, conforme pode ser observado na Tabela 2. Isso demonstra a estabilidade da distribuição dos parâmetros, embora os mesmos não apresentem normalidade multivariada. Essa informação indica que as estimações produzidas são confiáveis, ainda que os dados não sejam normais. De forma geral, os índices de ajuste desta análise apontam para a adequação da estrutura fatorial da BPP, corroborando os achados no Estudo 1, como pode ser observado na Tabela 3. Entretanto, esses índices indicaram que alguns dos fatores da BPP não se ajustam satisfatoriamente aos dados, especialmente os fatores RMM, RRO e DP, o que indica que há espaço para aprimoramento da BPP em alguns de seus fatores.

Tabela 2. Cargas fatoriais da BPP obtidas pelo MLE e indicadores das estimações em 200 reamostragens

\begin{tabular}{|c|c|c|c|c|c|}
\hline \multirow[t]{2}{*}{ Item } & \multicolumn{3}{|c|}{ MLE } & \multicolumn{2}{|c|}{ Booststrap } \\
\hline & $\mathrm{CB}^{*}$ & $\mathrm{CP}^{*}$ & $\mathrm{RC}^{*}$ & Média & EP* \\
\hline \multicolumn{6}{|c|}{ Responsabilidade social } \\
\hline Item 1 & 1,00 & 0,51 & & 1,00 & \\
\hline Item 2 & 0,91 & 0,45 & 6,27 & 0,93 & 0,18 \\
\hline Item 4 & 0,49 & 0,32 & 5,06 & 0,51 & 0,15 \\
\hline Item 5 & 0,55 & 0,34 & 5,30 & 0,55 & 0,14 \\
\hline Item 6 & 1,12 & 0,58 & 6,82 & 1,17 & 0,20 \\
\hline Item 7 & 0,62 & 0,39 & 5,79 & 0,64 & 0,12 \\
\hline \multicolumn{6}{|c|}{ Preocupação empática } \\
\hline Item 9 & 1,00 & 0,22 & & 1,00 & \\
\hline Item 11 & $-3,25$ & $-0,72$ & $-3,69$ & $-4,15$ & 3,39 \\
\hline Item 13 & $-3,21$ & $-0,59$ & $-3,81$ & $-3,83$ & 2,38 \\
\hline Item 15 & 1,59 & 0,37 & 3,56 & 1,86 & 1,14 \\
\hline \multicolumn{6}{|c|}{ Tomada de perspectiva } \\
\hline Item 8 & 1,00 & 0,39 & & 1,00 & \\
\hline Item 10 & $-0,96$ & $-0,56$ & $-6,38$ & $-0,98$ & 0,25 \\
\hline Item 12 & 0,91 & 0,36 & 5,25 & 0,91 & 0,19 \\
\hline Item 16 & $-1,14$ & $-0,61$ & $-6,50$ & $-1,18$ & 0,30 \\
\hline Item 18 & $-1,37$ & $-0,60$ & $-6,48$ & $-1,38$ & 0,24 \\
\hline \multicolumn{6}{|c|}{ Desconforto pessoal } \\
\hline Item 17 & 1,00 & 0,73 & & 1,00 & \\
\hline Item 19 & 0,66 & 0,72 & 10,25 & 0,65 & 0,09 \\
\hline Item 14 & $-0,56$ & $-0,59$ & $-10,15$ & $-0,55$ & 0,09 \\
\hline \multicolumn{6}{|c|}{ Raciocínio moral mútuo } \\
\hline Item 21 & 1,00 & 1,30 & & 1,00 & \\
\hline Item 22 & 0,16 & 0,15 & 4,52 & 0,16 & 0,04 \\
\hline Item 24 & 0,24 & 0,30 & 8,47 & 0,24 & 0,04 \\
\hline
\end{tabular}


Tabela 2. Cargas fatoriais da BPP obtidas pelo MLE e indicadores das estimações em 200 reamostragens

\begin{tabular}{|c|c|c|c|c|c|}
\hline \multirow[t]{2}{*}{ Item } & \multicolumn{3}{|c|}{ MLE } & \multicolumn{2}{|c|}{ Booststrap } \\
\hline & $\mathrm{CB}^{*}$ & $\mathrm{CP} *$ & $\mathrm{RC}^{*}$ & Média & EP* \\
\hline \multicolumn{6}{|c|}{ Raciocínio relacionado ao outro } \\
\hline Item 20 & 1,00 & 0,82 & & 1,00 & \\
\hline Item 23 & 0,79 & 0,77 & 22,23 & 0,78 & $0,0 c$ \\
\hline Item 25 & 0,99 & 0,90 & 27,21 & 0,99 & 0,0 \\
\hline \multicolumn{6}{|c|}{ Altruísmo auto-relatado } \\
\hline Item 26 & 1,00 & 0,76 & & 1,00 & \\
\hline Item 27 & 0,80 & 0,68 & 13,85 & 0,80 & 0,05 \\
\hline Item 28 & 1,08 & 0,62 & 12,73 & 1,09 & 0,10 \\
\hline Item 29 & 1,03 & 0,50 & 10,33 & 1,04 & 0,12 \\
\hline Item 30 & 1,06 & 0,71 & 14,24 & 1,07 & 0,07 \\
\hline
\end{tabular}

*CB - Coeficiente Bruto da estimação MLE; CP - Coeficiente Padronizado da estimação MLE; RC - Razão Crítica da estimação MLE; EP Desvio Padrão da estimação nas amostras Bootstrap

Tabela 3. Índices de Ajuste para Cada um dos Fatores de Primeira Ordem da BPP

\begin{tabular}{lccc}
\hline Fatores & GFI & CFI & RMSEA \\
\hline Responsabilidade social & 0,98 & 0,87 & 0,08 \\
Preocupação empática & 0,98 & 0,89 & 0,14 \\
Tomada de perspectiva & 0,99 & 0,96 & 0,07 \\
Desconforto pessoal & 0,96 & 0,90 & 0,24 \\
Raciocínio moral mútuo & 0,92 & 0,61 & 0,38 \\
Raciocínio relacionado ao outro & 0,93 & 0,89 & 0,33 \\
Altruísmo auto-relatado & 0,98 & 0,96 & 0,10 \\
\hline
\end{tabular}

Como forma de teste da estrutura de segunda ordem, foi realizada uma análise fatorial confirmatória envolvendo apenas as dimensões de segunda ordem que agrupariam os sete fatores da BPP. Conforme Penner e cols. (1995), as duas dimensões estão assim organizadas: prestatividade agrupa os fatores AAR e DP; e empatia orientada ao outro agrupa os fatores RS, PE, TP, RMM e RRO. Essa análise indicou que a estrutura de duas dimensões é razoavelmente satisfatória $\quad[\mathrm{GFI}=0,93 ; \quad \mathrm{CFI}=0,84 ; \quad \mathrm{RMSEA}=0,13$ $\left(\mathrm{IC}_{90}=0,11\right.$ a 0,15$\left.)\right]$. Entretanto, apesar das dimensões de segunda ordem não terem sido replicadas de maneira totalmente satisfatória, os fatores de primeira ordem se aproximam consideravelmente dos dados relatados nos estudos originais, o que indica que a BPP pode ser utilizada no contexto de investigação do comportamento prosocial. Também foi identificada uma diferença de sexo quanto à PE, em relação à qual os homens tiveram uma média maior $(M=1,98$; $D P=0,66)$ do que a das mulheres $(M=1,76 ; D P=0,57)$, $F(1,511)=16,98 ; p<0,0001 ; \eta_{\mathrm{P}}{ }^{2}=0,03$, e também em relação à $\mathrm{DP}$, sendo que homens tiveram uma média menor $(M=2,26 ; D P=0,81)$ do que a das mulheres Psico-USF, Bragança Paulista, v. 18, n. 3, p. 455-468, set/dez 2013
$(M=2,40 ; \quad D P=0,75), \quad F \quad(1, \quad 511)=3,97 ; \quad p=0,047$, $\eta_{\mathrm{p}}{ }^{2}=0,008$.

Os dados da análise fatorial confirmatória realçaram os problemas psicométricos observados no Estudo 1, mesmo que tenha sido possível replicar parcialmente a estrutura original e identificar índices psicométricos aceitáveis (Penner \& cols., 1995). Embora os dados não tenham apresentado normalidade multivariada, o procedimento de bootstrapping revelou que havia estabilidade na distribuição dos parâmetros estimados. A falta de normalidade dos dados era esperada, considerando os elementos de desejabilidade social diretamente associados à temática avaliada pela BPP - por se tratar de uma medida explícita e de autorrelato sobre a prosocialidade auto percebida pelos indivíduos - é coerente que os dados tenham se distribuído de maneira não uniforme e se concentrado nas extremidades da escala (Nosek \& cols., 2011; Paulhus, 1984). Em contraponto a isso, entretanto, os resultados do bootstrapping demonstram que a falta de normalidade não influenciou a estimação dos parâmetros, indicando que tais estimações são confiáveis. Considerando a 
magnitude das cargas fatoriais, pode-se concluir que a estrutura de primeira ordem é adequada para o uso da BPP em diferentes contextos de investigação. Quanto às diferenças de sexo, foram encontrados resultados semelhantes aos do Estudo 1, incluindo as discrepâncias com a literatura prévia (Sampaio \& Menezes, 2011). A seguir, discutiremos os dados dos dois estudos enfatizando aspectos estatísticos que podem explicar a relevância e a replicabilidade $\mathrm{da}$ diferença encontrada.

\section{Discussão geral}

As evidências de uma organização dos sete fatores de primeira ordem em duas dimensões de segunda ordem foram frágeis no presente trabalho, embora os índices psicométricos identificados sejam aceitáveis. Tal resultado pode ser em razão da estratégia pouco ortodoxa de construção da bateria, conforme relatado por Penner \& cols. (1995), na área de desenvolvimento de medidas em psicologia social e da personalidade. $\mathrm{O}$ procedimento dos autores de selecionar itens e fatores de instrumentos já apresentados na literatura e que avaliavam construtos conceitualmente correlatos ao comportamento prosocial, e a posterior análise sistemática de redução de itens, até se chegar à estrutura que foi testada neste trabalho, pode ter ocasionado vieses de análise, como, por exemplo, a dificuldade na replicação das duas dimensões de segunda ordem. De qualquer sorte, como já apresentado anteriormente, a BPP tem demonstrado uma boa capacidade preditiva de diferentes comportamentos prosociais, sejam de curto ou longo prazo, o que dá indícios de sua validade e utilidade para o contexto de pesquisa em psicologia social (Finkelstein \& cols., 2005; Penner \& Finkelstein, 1998; Penner, 2002; Perugini \& cols., 2011; Rabelo \& cols., 2012).

Quanto às diferenças de sexo (Sampaio \& Menezes, 2011), encontrou-se um padrão muito semelhante nos dois estudos: homens demonstraram maiores níveis de PE e menores níveis de DP do que mulheres. Entretanto, os tamanhos de efeito associados a essas diferenças são baixos (Levine \& Hullett, 2002), apesar das diferenças serem estatisticamente significativas. Considerando o tamanho das nossas amostras, a probabilidade de se identificar alguma relação que atinja a significância é maior, embora tais diferenças não sejam necessariamente relevantes (Cohen, 1994; Wagenmakers \& cols., 2011), e os dados relacionados com o tamanho de efeito apresentados aqui indicam que as diferenças não são robustas. A comparação, nesse aspecto, com estudos anteriores é comprometida pela ausência de relato do tamanho de efeito (Sampaio \& Menezes, 2011), algo ainda comum na psicologia (Kelley \& Preacher, 2012).

Também foi possível encontrar evidências a favor da estabilidade transcultural da personalidade prosocial, mensurada por meio da BPP, já que os dados identificados no presente estudo guardam consistência com aqueles obtidos em amostras de outros países (Kulik, 2007; Levine \& cols., 2001; Penner, 2002; Penner \& cols., 2005; Perugini \& cols., 2011; Rabelo \& cols., 2012). Entretanto, antes que se possa fazer uma discussão sobre diferenças regionais ou culturais, é necessário que estudos futuros investiguem não apenas se existem diferenças, mas também o quão robustas elas são, já que isso afeta a probabilidade de replicação de tais diferenças em outros estudos e a própria relevância teórica, empírica e prática de tais evidências.

O presente estudo possui algumas limitações. Dentre outras, convém destacar duas. A primeira diz respeito à ausência de dados para o teste de validade convergente com outras medidas, como é o caso da Interpersonal Reactivity Index (Sampaio \& Menezes, 2011) ou do fator de agradabilidade (sociabilidade) de alguma das baterias do Big Five, já com evidências de validade no país. À guisa de desenvolvimento continuado desta versão adaptada da BPP, seria relevante a criação de uma rede nomológica por meio do teste de convergência com outros instrumentos que avaliam construtos correlatos àqueles mensurados pela BPP. A rede nomológica é um conjunto de princípios para o teste de validade de instrumentos de medida que consideram, entre outros aspectos, o estabelecimento de redes de associação entre: (a) propriedades ou quantidades observadas entre si; (b) construtos teóricos com variáveis observadas; e (c) construtos teóricos diferentes entre si (Cronbach \& Meehl, 1955). Com base nesses princípios, é recomendado que o desenvolvimento de qualquer instrumento de medida seja submetido a uma rede de associações com outros instrumentos, o que permita aferir indicadores de convergência, divergência e de predição de medidas de diversos construtos.

Um segundo aspecto diz respeito ao fato de a BPP ser uma medida explícita de autorrelato. Essa medida é sobre um tema que envolve alta desejabilidade social e depende das capacidades introspectivas e de recuperação de informações limitadas que os participantes dispõem no momento da pesquisa, uma crítica que têm sido feita às medidas baseadas em autorrelato desde os anos 1970 (Nisbett \& Wilson, 1977; Nosek \& cols., 2011; Paulhus, 1984). Neste sentido, há necessidade de explorar alternativas às medidas explícitas de autorrelato sobre o comportamento prosocial. Uma possibilidade é o desenvolvimento de medidas implícitas que visem 
medir diferenças individuais (Greenwald, McGhee \& Schwartz, 1998) na prosocialidade, como foi feito no estudo de Perugini e cols. (2011). A comparação entre medidas implícitas e explícitas de prosocialidade pode demonstrar o valor diferencial que cada uma pode oferecer para predizer diferentes tipos de comportamento prosocial. Perugini e cols. demonstraram em seu estudo, por exemplo, que a personalidade prosocial predizia melhor comportamentos prosociais de caráter mais deliberado e controlado, enquanto a medida implícita de prosocialidade predizia melhor comportamentos prosociais de caráter mais automático e espontâneo. Portanto, o investimento no desenvolvimento de medidas implícitas é uma ação relevante para o avanço dos estudos no campo das diferenças individuais do comportamento prosocial.

Ainda que existam lacunas a serem resolvidas na área, por meio da continuidade da linha de investigação, considera-se que o objetivo de adaptar e produzir evidências de validade da BPP comparáveis aos dados originais de Penner e cols. (1995) para o contexto brasileiro foi alcançado e que este instrumento apresenta qualidades psicométricas aceitáveis, habilitando o seu uso para a pesquisa desse tipo de comportamento. A versão produzida neste trabalho pode ser usada para investigar esse tipo de comportamento dos brasileiros e enriquecer o conhecimento que possuímos acerca do papel das diferenças individuais na prosocialidade, para a predição do comportamento prosocial e para o estudo de modelos interativos de antecedentes da prosocialidade, como é o caso de modelos que consideram o efeito moderador das diferenças individuais entre fatores situacionais e $\mathrm{O}$ comportamento (Perugini \& Prestwich, 2007).

\section{Referências}

Beú, N. B. (2010). Motivaçẽes para o voluntariado empresarial: desenvolvimento de um instrumento de medida (Dissertação de mestrado não publicada). Universidade de Brasília, Brasilia.

Byrne, B. M. (2010). Structural equation modeling with AMOS: basic concepts, applications, and programming. Structural Equation Modeling (2nd ed., Vol. 22). Nova Iorque: Routledge/Taylor \& Francis.

Caprara, G. V., Alessandri, G. \& Eisenberg, N. (2012). Prosociality: the contribution of traits, values, and self-efficacy beliefs. Journal of Personality and Social Psychology, 102(6), 1289-1303.

Carlo, G., Eisenberg, N., Troyer, D., Switzer, G. \& Speer, A. L. (1991). The altruistic personality: in what contexts is it apparent? Journal of Personality and Social Psychology, 61, 450-458.

Cohen, J. (1994). The earth is round $(\mathrm{p}<.05)$. American Psychologist, 49, 997-1003.

Cronbach, L. J. \& Meehl, P. E. (1955). Construct validity in psychological tests. Psychological Review, 52, 281-302.

Dovidio, J. F., Piliavin, J. A., Schroeder, D. A. \& Penner, L. A. (2006). The social psychology of prosocial behavior. Group. Nova Iorque: Lawrence Erlbaum.

EFS survey. (2012). [Computer Software] KoelnHuerth: QuestBack AG.

Eisenberg, N., Guthrie, I. K., Cumberland, A., Murphy, B. C., Shepard, S. A., Zhou, Q. \& Carlo, G. (2002). Prosocial development in early adulthood: a longitudinal study. Journal of Personality and Social Psychology, 82(6), 993-1006.

Finkelstein, M. A., Penner, L. A. \& Brannick, M. T. (2005). Motive, role identity, and prosocial personality as predictors of volunteer activity. Social Behavior and Personality, 33(4), 403-418.

Graziano, W. G., Habashi, M. M., Sheese, B. E. \& Tobin, R. M. (2007). Agreeableness, empathy, and helping: a person x situation perspective. Journal of Personality and Social Psychology, 93(4), 583-99.

Greenwald, A. G., McGhee, D. E. \& Schwartz, J. L. (1998). Measuring individual differences in implicit cognition: the implicit association test. Journal of Personality and Social Psychology, 74(6), 1464-1480.

Hambleton, R. K. \& Zenisky, A. L. (2011). Translating and adapting tests for cross-cultural assessments. Em D. Matsumoto \& F. van de Vijver (Eds.). Cross-cultural research methods in psychology (pp. 46-74). Nova Iorque: Cambridge University Press.

Kelley, K., \& Preacher, K. J. (2012). On effect size. Psychological methods, 17(2), 137-52.

Kerekes, Z., Bereczkei, T. \& Birkas, B. (2010). The presence of others, prosocial traits, machiavellianism: a personality $\times$ situation approach. Social Psychology, 41(4).

Kulik, L. (2007). Predicting responses to volunteering among adolescents in Israel: the contribution of personal and situational variables. VOLUNTAS: International Journal of Voluntary and Nonprofit Organizations, 18(1), 35-54.

LeBel, E. P. \& Paunonen, S. V. (2011). Sexy but often unreliable: The impact of unreliability on the replicability of experimental findings with implicit measures. Personality and Social Psychology Bulletin, 37(4), 570-583.

Levine, T. R. \& Hullett, C. R. (2002). Eta squared, partial eta squared and the misreporting of effect size in communication research. Human Communication Research, 28, 612-625. 
Levine, R. V., Norenzayan, A. \& Philbrick, K. (2001). Cross-cultural differences in helping strangers. Journal of Cross-Cultural Psychology, 32(5), 543-560.

Nisbett, R. E., \& Wilson, T. D. (1977). Telling more than we can know: verbal reports on mental processes. Psychological Review, 84, 231-259.

Nosek, B. A., Hawkins, C. B. \& Frazier, R. S. (2011). Implicit social cognition: From measures to mechanisms. Trends in Cognitive Sciences, 15(4), 152159.

Paulhus, D. L. (1984). Two-component models of socially desirable responding. Journal of Personality and Social Psychology, 46(3), 598-609.

Penner, L. A. (n.d.). User's guide to the Prosocial Personality Battery (PSB) [Arquivo de texto]. Acesso em: 04/jun/2013 http://www.med.wayne.edu/fam/faculty/pdfs/30 itempsbkey.pdf

Penner, L. A. (2002). Dispositional and organizational influences on sustained volunteerism: An interactionist perspective. Journal of Social Issues, 58(3), 447-467.

Penner, L. A., Dovidio, J. F., Piliavin, J. A. \& Schroeder, D. A. (2005). Prosocial behavior: multilevel perspectives. Annual Review of Psychology, 56(56), 365-92.

Penner, L. A. \& Finkelstein, M. A. (1998). Dispositional and structural determinants of volunteerism. Journal of Personality and Social Psychology, 74(2), 525-537.

Penner, L. A., Fritzsche, B. A., Craiger, J. P. \& Freifeld, T. R. (1995). Measuring the prosocial personality. Em J. Butcher \& C. D. Spielberger (Eds.). Advances in Personality Assessment (Vol. 10, pp. 147-163). Hillsdale, NJ: Erlbaum.

Penner, L. A. \& Orom, H. (2010). Enduring goodness: A person-by-situation perspective on prosocial behavior. Em M. Mikulincer \& P. R. Shaver (Eds.), Prosocial motives, emotions, and behavior: the better angels of our nature. (pp. 55-72). Washington: APA.
Perugini, M., Conner, M. \& O’Gorman, R. (2011). Automatic activation of individual differences: A test of the gatekeeper model in the domain of spontaneous helping. European Journal of Personality, 25, 465-476.

Perugini, M. \& Prestwich, A. (2007). The gatekeeper: Individual differences are key in the chain from perception to behaviour. European Journal of Personality, 21, 303-317.

Pilati, R. \& Laros, J. A. (2007). Modelos de equações estruturais em psicologia: Conceitos e aplicações. Psicologia: Teoria e Pesquisa, 23, 205-216.

Rabelo, A. L. A., Hees, M. A. G. \& Pilati, R. (2012). A moderação da prosocialidade entre o priming e a intenção de gentileza. Psico (PUCRS. Online), 43, 163-173.

Sampaio, L. R. \& Menezes, I. G. (2011). Estudos sobre a dimensionalidade da empatia: Tradução e adaptação do Interpersonal Reactivity Index (IRI). Psico (PUCRS. Online), 42(1), 67-76.

Van Lange, P. A., Otten, W., De Bruin, E. M. \& Joireman, J. A. (1997). Development of prosocial, individualistic, and competitive orientations: Theory and preliminary evidence. Journal of Personality and Social Psychology, 73(4), 733-46.

Wagenmakers, E. J. J., Wetzels, R., Borsboom, D., van der Maas, H. L. J. \& Maas, H. L. J. Van Der. (2011). Why psychologists must change the way they analyze their data: the case of psi: Comment on Bem (2011). Journal of Personality and Social Psychology, 100, 426-32.
Recebido em 05/12/2012

Revisado em 19/10/2013

Aprovado em 23/10/2013 
Anexo 1 - Itens da Bateria de Personalidade Prosocial (BPP)

1. Quando as pessoas são más comigo, eu me sinto desobrigado de tratá-las bem.

2. Eu me sentiria menos incomodado ao deixar lixo em um parque sujo do que em um parque limpo.

3. Não importa o que uma pessoa fez conosco, não há desculpa para tirar vantagem desta pessoa.

4. Com a pressão por tirar boas notas e com o aumento das pessoas que colam na escola hoje em dia, o indivíduo que ocasionalmente cola não tem muita culpa.

5. Quando estamos doentes e nos sentindo mal, ficar preocupado com o modo como agimos não faz muito sentido.

6. Se eu quebrei uma máquina por não operá-la corretamente, eu me sentiria menos culpado se descobrisse que ela já estava quebrada antes de tê-la usado.

7. Quando temos um trabalho para fazer, é impossível conseguir contemplar os interesses e necessidades das outras pessoas.

8. Às vezes eu acho difícil ver as coisas do ponto de vista de outra pessoa.

9. Quando eu vejo alguém em desvantagem, me sinto um pouco protetor desta pessoa.

10. Às vezes eu tento entender melhor meus amigos ao imaginar como as coisas são do ponto de vista deles.

11. A miséria dos outros geralmente não me incomoda muito.

12. Se eu tenho certeza de algo, não gosto de perder tempo ouvindo o argumento dos outros.

13. Quando vejo alguém sendo maltratado, às vezes não sinto muita pena dessa pessoa.

14. Geralmente sou muito eficiente ao lidar com emergências.

15. Às vezes me sinto tocado pelo que vejo acontecer.

16. Eu acredito que há dois lados em cada questão e tento analisar esses dois lados.

17. Tenho tendência a perder o controle durante emergências.

18. Quando estou chateado com alguém, geralmente procuro me colocar em seu lugar.

19. Quando vejo alguém que precisa muito de ajuda em uma situação de emergência, eu fico perdido.

20. Minhas decisões são geralmente baseadas na minha preocupação com outras pessoas.

21. Minhas decisões são geralmente baseadas no que é mais justo e na maneira mais correta de agir.

22. Eu escolho alternativas com a intenção de satisfazer as necessidades de todo mundo.

23. Eu escolho um modo de agir que permite ajudar outras pessoas ao máximo.

24. Eu escolho um modo de agir que considera os direitos de todas as pessoas envolvidas.

25. Minhas decisões são geralmente baseadas no bem-estar dos outros.

26. Eu já ajudei a carregar os pertences de um estranho (ex. livros, pacotes, etc.).

27. Eu permiti que alguém passasse na minha frente em uma fila (ex. supermercado, fotocopiadora, etc.).

28. Eu já emprestei um item de algum valor para um vizinho que não conhecia bem (ex. ferramentas, um prato, etc.).

29. Eu já me ofereci para cuidar dos animais de estimação ou crianças de um vizinho sem ser remunerado por isso.

30. Eu já me ofereci para ajudar um deficiente ou idoso desconhecido a atravessar a rua. 
Nota dos autores:

Financiamento do CNPq por meio de bolsa de iniciação científica (PROIC-UnB) do primeiro autor e bolsa produtividade (PQ) do segundo autor e do Edital Universal do CNPq (Processo 470223/2011-1).

Sobre os autores:

André L. A. Rabelo é aluno de mestrado no programa de pós-graduação em Psicologia Social, do Trabalho e das Organizações (PSTO) da Universidade de Brasília (UnB).

Ronaldo Pilati possui Doutorado em Psicologia pela Universidade de Brasília. É professor adjunto do Departamento de Psicologia Social e do Trabalho (PST) e do Programa de Pós-Graduação em Psicologia Social, do Trabalho e das Organizações (PSTO) da UnB.

Contato com os autores:

André Luiz Alves Rabelo

Campus Universitário Darcy Ribeiro

Departamento de Psicologia Social e do Trabalho

Sala AT-013, ICC/Sul, Asa Norte

CEP 70910-900 - Brasília/DF, Brasil

Fone/Fax: (61) 31076828

E-mail: andreluiz.arabelo@gmail.com 